\title{
Timed-Release Certificateless Encryption
}

\author{
Toru Oshikiri \\ Graduate School of Engineering \\ Tokyo Denki University \\ Tokyo, Japan
}

\author{
Taiichi Saito \\ Tokyo Denki University \\ Tokyo, Japan
}

\begin{abstract}
Timed-Release Encryption(TRE) is an encryption mechanism that allows a receiver to decrypt a ciphertext only after the time that a sender designates. In this paper, we propose the notion of Timed-Release Certificateless Encryption(TRCLE), and define its security models. We also show a generic construction of TRCLE from Public-Key Encryption(PKE), IdentityBased Encryption(IBE) and one-time signature, and prove that the constructed scheme achieves the security we defined.
\end{abstract}

Keywords-timed-release encryption, identity-based encryption, one-time signature

\section{INTRODUCTION}

This paper introduces the notion of Timed-Release Certificateless Encryption (TRCLE). TRCLE is a variant of TimedRelease Encryption (TRE) [1] [2], in which a sender can generate a ciphertext designating a time to decrypt it, and a receiver can decrypt the ciphertext only after the designated time.

A TRCLE system consists of a key generation center (KGC), a time server (TS), senders and receivers. The KGC helps a receiver to generate a decryption key corresponding to the ID and public key of a receiver. The TS periodically broadcasts a time signal corresponding to the current time. A sender encrypts a message using an ID and a public key of a receiver and a time after which the ciphertext could be decrypted. The receiver decrypts the ciphertext using the decryption key and the time signal corresponding to the time designated by the sender. The TRCLE system does not allow the KGC to obtain the decryption key of receiver, and then it allows only the receiver to decrypt the ciphertext only after the designated time.

The decryption key consists of two keys, a partial secret key and user secret key. Since the former is generated by the $\mathrm{KGC}$ and the user but the latter only by the user, the KGC does not know the whole decryption key and cannot decrypt ciphertext.

\section{APPLICATION}

TRCLE has an application to online "sealed-bid auction" in online community in which each registered user has an ID. In the auction system, every user can become auctioneer by publicizing his ID and public key. Each bidder encrypts his price by using the auctioneer's ID and public key and submits the ciphertext as sealed-bid. The auctioneer can decrypt all bids only after the pre-determined closing time. In the sealedbid auction based on TRCLE, each user determines whether he trusts the auctioneer of ID and attends the auction by checking the reputations and the transaction records in the past auctions organized by the user of ID. Every user easily starts a sealedbid auction based on TRCLE, since it does not requires heavy infrastructure linking public keys to ID such as Public-Key Infrastructure (PKI)

\section{RELATED WORKS}

There is another variant of TRE, Timed-Release IdentityBased Encryption (TRIBE) [3] [4]. In TRIBE, a user can decrypt a ciphertext only when the user has the receiver's secret key and the time signal generated by TS. Then, if the receiver does not have the time signal or the TS does not have the secret key, they cannot decrypt the ciphertext. In [3], two security models of TRIBE are defined. One is security against malicious receiver, IND-ID-CCA $\mathrm{CR}_{\mathrm{R}}$ security. The other is security against malicious TS, IND-ID-CCA TS $_{\text {security. A }}$ generic construction of TRIBE that achieves the security is also shown in [3]. It is a combination of two IBE schemes and a one-time signature schemes, based on "Parallel Encryption" by Dodis-Katz [5], and the security is proved in the standard model.

TRCLE has an advantage over TRIBE in that a compromised KGC cannot decrypt any ciphertext since the key generation process is split between the KGC and the user. Then we discuss only security against malicious $\mathrm{KGC}$ in this paper. The other security is proved in almost the same way as in TRIBE.

TRCLE can be considered also a variant of Certificateless Encryption (CLE) [6] having the mechanism of TRE. In CLE, the decryption key is partially determined by KGC

\section{CONTRIBUTIONS}

In this paper, we introduce timed-release certificateless encryption (TRCLE) and define its security models including security against malicious KGC, Mal.KGC security. We also present a generic construction of TRCLE. It is a combination of a Public-Key Encryption(PKE) scheme, two Identity-Based Encryption(IBE) schemes and a one-time signature schemes, also based on "Parallel Encryption". We see that if the primitive PKE scheme is indistinguishability against adaptive chosen ciphertext attacks(IND-CCA) secure and the primitive one-time signature scheme is one-time strong existential unforgeability against chosen message attacks(OT-sEUF-CMA) secure, then the constructed TRCLE scheme is Mal.KGC secure in the standard model. 


\section{PRELIMINARIES}

In this section, we review public-key encryption (PKE), identity-based encryption (IBE) and one-time signature, which we use later.

\section{A. Public-Key Encryption}

Let $\lambda$ be a security parameter. An public-key encryption scheme $\mathcal{P} \mathcal{K} \mathcal{E}$ [7] consists of three probabilistic polynomialtime algorithms $\mathcal{P} \mathcal{K} \mathcal{E}=($ PKE.Gen, PKE.Enc, PKE.Dec $)$. The key generation algorithm PKE.Gen takes $\lambda$ as input, and outputs a public key $p k$ and a secret key $s k$. The encryption algorithm PKE.Enc takes $p k$, a message $m$ as inputs, and outputs a ciphertext $c$. The decryption algorithm PKE.Dec takes a secret key $s k$ and a ciphertext $c$ as inputs, and outputs the plaintext $m^{\prime}$ or $\perp$. These algorithms are assumed to satisfy that if $(p k, s k)$ $=\operatorname{PKE} \cdot \operatorname{Gen}(\lambda)$ then PKE.Dec $(s k, \operatorname{PKE} \cdot \operatorname{Enc}(p k, m))=m$ for any $m$.

1) IND-CCA Security: We review a standard security notion for PKE: indistinguishability against adaptive chosen ciphertext attacks (IND-CCA) security. We here describe the IND-CCA security for PKE scheme $\mathcal{P} \mathcal{K} \mathcal{E}$ based on the following IND-CCA game between a challenger $\mathcal{C}$ and an adversary $\mathcal{A}$.

\section{Setup \\ Phase1 \\ $\mathcal{C}$ runs $(p k, s k) \leftarrow$ PKE.Gen $(\lambda) . \mathcal{C}$ sends $p k$ to $\mathcal{A}$ and keeps $s k$ secret. \\ $\mathcal{A}$ can adaptively issue decryption queries $c . \mathcal{C}$ responds to a decryption query $c$ by running $m^{\prime}=$ PKE.Dec $(s k, c)$, and returning $m^{\prime}$ to $\mathcal{A}$. \\ Challenge \\ $\mathcal{A}$ sends two messages $m_{0}, m_{1}$ such that $\left|m_{0}\right|=\left|m_{1}\right|$ to $\mathcal{C}$. $\mathcal{C}$ randomly chooses $b \in$ $\{0,1\}$ and sends a challenge ciphertext $c^{*}=$ PKE.Enc $\left(p k, m_{b}\right)$ to $\mathcal{A}$.}

Phase2

$\mathcal{A}$ can adaptively issue decryption queries $c$ in the same way as in Phase1 except that the decryption queries $c$ must differ from the challenge ciphertext $c^{*}$.

Guess

$\mathcal{A}$ outputs a guess $b^{\prime} \in\{0,1\}$ and wins if $b=b^{\prime}$.

We define an advantage of $\mathcal{A}$ in the IND-CCA game as $A d v_{\mathcal{P} \mathcal{K}, \mathcal{E}, \mathcal{A}}^{\text {IND-CCA }}(\lambda)=\left|\operatorname{Pr}\left[b=b^{\prime}\right]-\frac{1}{2}\right|$, in which the probability is taken over the random coins used by $\mathcal{C}$ and $\mathcal{A}$. We say that the PKE scheme $\mathcal{P} \mathcal{K} \mathcal{E}$ is IND-CCA secure if, for any probabilistic polynomial-time adversary $\mathcal{A}$, the function $\operatorname{Adv}_{\mathcal{P} \mathcal{K} \mathcal{E}, \mathcal{A}}{ }^{\mathrm{IND}}(\lambda)$ is negligible in $\lambda$.

\section{B. Identity-Based Encryption}

Let $\lambda$ be a security parameter. An identity-based encryption scheme $\mathcal{I B E}$ [8] consists of four probabilistic polynomial-time algorithms $\mathcal{I} \mathcal{B E}=$ (IBE.Setup, IBE.Ext, IBE.Enc, IBE.Dec). The setup algorithm IBE.Setup takes $\lambda$ as input, and outputs a public parameter params and a master secret key msk. The extract algorithm IBE.Ext takes params, msk, and an identity ID as inputs, and outputs a decryption key $d_{\mathrm{ID}}$. The encryption algorithm IBE.Enc takes params, ID, a message $m$ as inputs, and outputs a ciphertext $c$. The decryption algorithm IBE.Dec takes params, a decryption key $d_{\mathrm{ID}}$ and a ciphertext $c$ as inputs, and outputs the plaintext $m^{\prime}$ or $\perp$. These algorithms are assumed to satisfy that if (params, msk) = $\operatorname{IBE} \cdot \operatorname{Setup}(\lambda)$ and $d_{\mathrm{ID}}=\operatorname{IBE} . \operatorname{Ext}($ params, $m s k$, ID) then IBE.Dec $\left(\right.$ params, $d_{\mathrm{ID}}$, IBE.Enc $($ params $\left., \mathrm{ID}, m)\right)=m$ for any $m$.

1) IND-ID-CCA Security: We review a standard security notion for IBE: indistinguishability against adaptive identity and chosen ciphertext attacks (IND-ID-CCA) security [9]. We here describe the IND-ID-CCA security for IBE scheme $\mathcal{I B E}$ based on the following IND-ID-CCA game between a challenger $\mathcal{C}$ and an adversary $\mathcal{A}$.

Setup

Phase1

$\mathcal{C}$ runs $($ params, msk $) \leftarrow \operatorname{IBE} . \operatorname{Setup}(\lambda) . \mathcal{C}$ sends params to $\mathcal{A}$ and keeps $m s k$ secret.

$\mathcal{A}$ can adaptively issue extraction queries ID and decryption queries $(\mathrm{ID}, c) . \mathcal{C}$ responds to an extraction query ID by running $d_{\mathrm{ID}}=$ IBE.Ext (params, msk, ID) and returning $d_{\mathrm{ID}}$ to $\mathcal{A}$. $\mathcal{C}$ responds to a decryption query $(\mathrm{ID}, c)$ by running $d_{\mathrm{ID}}=\mathrm{IBE} . \operatorname{Ext}($ params, $m s k, \mathrm{ID})$ and $m^{\prime}=\mathrm{IBE} . \operatorname{Dec}\left(\right.$ params $\left., d_{\mathrm{ID}}, c\right)$, and returning $m^{\prime}$ to $\mathcal{A}$.

\section{Challenge}

$\mathcal{A}$ sends two messages $m_{0}, m_{1}$ such that $\left|m_{0}\right|=$ $\left|m_{1}\right|$, and an identity to be challenged ID* $^{*}$ to $\mathcal{C}$. The challenge identity ID* must differ from any ID issued as extraction query in Phase1. $\mathcal{C}$ randomly chooses $b \in\{0,1\}$ and sends a challenge ciphertext $c^{*}=\operatorname{IBE}$. Enc $\left(\right.$ params, ID $\left.{ }^{*}, m_{b}\right)$ to $\mathcal{A}$.

Phase2

$\mathcal{A}$ can adaptively issue extraction queries ID and decryption queries $(I D, c)$ in the same way as in Phase1 except that the extraction queries ID must differ from the challenge identity ID*, and decryption queries (ID, $c$ ) must differ from the pair $\left(\mathrm{ID}^{*}, c^{*}\right)$.

Guess

$\mathcal{A}$ outputs a guess $b^{\prime} \in\{0,1\}$ and wins if $b=b^{\prime}$.

We define an advantage of $\mathcal{A}$ in the IND-ID-CCA game as $A d v_{\mathcal{I} \mathcal{B} \mathcal{E}, \mathcal{A}}^{\mathrm{IND}}(\lambda)=\left|\operatorname{Pr}\left[b=b^{\prime}\right]-\frac{1}{2}\right|$, in which the probability is taken over the random coins used by $\mathcal{C}$ and $\mathcal{A}$. We say that the IBE scheme $\mathcal{I B E}$ is IND-ID-CCA secure if, for any probabilistic polynomial-time adversary $\mathcal{A}$, the function $A d v_{\mathcal{I} \mathcal{B} \mathcal{E}, \mathcal{A}}^{\mathrm{ND}}(\lambda)$ is negligible in $\lambda$.

\section{One-time Signature}

Let $\lambda$ be a security parameter. $\mathcal{A}$ signature scheme $\mathcal{S I G}$ consists of three probabilistic polynomial-time algorithms $\mathcal{S I G}=$ (SigGen, Sign, Verify). The key generation algorithm SigGen takes $\lambda$ as input, and outputs a signing key $s k$ and a verification key $v k$. The signing algorithm Sign takes $s k$ and a message $m$ as inputs, and outputs a signature $\sigma$. The verification algorithm Verify takes $v k$, a message $m$, and a signature $\sigma$ as inputs, and outputs accept or reject. These 
algorithms are assumed to satisfy that if $(s k, v k)=\operatorname{SigGen}(\lambda)$ then $\operatorname{Verify}(v k, m, \operatorname{Sign}(s k, m))=$ accept for any $m$.

1) OT-sEUF-CMA Security: We review a security notion for one-time signature scheme: one-time strong existential unforgeability against chosen message attacks (OT-sEUFCMA) security [10]. We here describe the OT-sEUF-CMA security for signature scheme $\mathcal{S I G}$ based on the following OTsEUF-CMA game between a challenger $\mathcal{C}$ and an adversary $\mathcal{A}$.

\section{Setup \\ $\mathcal{C}$ runs the $(s k, v k) \leftarrow \operatorname{SigGen}(\lambda)$. $\mathcal{C}$ sends $v k$ to $\mathcal{A}$ and keeps $s k$ secret. \\ Query \\ $\mathcal{A}$ can issue a signing query $m$ to $\mathcal{C}$ only once. $\mathcal{C}$ responds to the singing query $m$ by running $\sigma=\operatorname{Sign}(v k, m)$ and returning $\sigma$ to $\mathcal{A}$. \\ Forge

$$
\mathcal{A} \text { outputs a pair }\left(m^{*}, \sigma^{*}\right) \text {. }
$$

We define the advantage of $\mathcal{A}$ in the OT-sEUF-CMA game as $\operatorname{Adv}_{\mathcal{S} \mathcal{I} \mathcal{G}, \mathcal{A}}^{\text {OT-sEUF-CMA }}(\lambda)=\operatorname{Pr}\left[\operatorname{Verify}\left(v k, m^{*}, \sigma^{*}\right)=\operatorname{accept} \wedge\right.$ $\left.(m, \sigma) \neq\left(m^{*}, \sigma^{*}\right)\right]$, in which the probability is taken over the random coins used by $\mathcal{C}$ and $\mathcal{A}$. We say that the signature scheme $\mathcal{S I G}$ is OT-sEUF-CMA secure if, for any probabilistic polynomial-time adversary $\mathcal{A}$, the function $\operatorname{Adv}_{\mathcal{S} \mathcal{I} \mathcal{G}, \mathcal{A}}^{\mathrm{OT}}{ }^{\mathrm{T}} \mathrm{AU}(\lambda)$ is negligible in $\lambda$.

\section{TIMED-RELEASE CERTIFICATELESS ENCRYPTION(TRCLE)}

In this section, we introduce timed-release certificateless encryption(TRCLE) scheme and define its security models.

A TRCLE system consists of a key generation center (KGC), a time server (TS), senders and receivers. The KGC helps a receiver to generate a partial secret key corresponding to an ID of the receiver. The TS periodically broadcasts a time signal corresponding to the current time. A sender encrypts a message using the ID and public key of a receiver, and a time after which the ciphertext could be decrypted. The receiver decrypts the ciphertext using the partial secret key, the user secret key and the time signal corresponding to the time designated by the sender.

Let $\lambda$ be a security parameter. An timed-release certificateless encryption scheme $\mathcal{T} \mathcal{R C} \mathcal{L} \mathcal{E}$ consists of seven probabilistic polynomial-time algorithms $\mathcal{T} \mathcal{R C} \mathcal{L} \mathcal{E}=(\mathrm{KGC}$ Setup, TS_Setup, PartialKeyGen, UserKeyGen, Release, Encrypt, Decrypt). The key generation center's setup algorithm KGC_Setup takes $\lambda$ as input, and outputs a public parameter params and a master secret key msk. The time server's setup algorithm TS_Setup takes $\lambda$ as input, and outputs a public key tpk and the corresponding secret key tsk. The partial secret key generation algorithm PartialKeyGen takes params, msk and ID as input, and outputs a partial secret key $p s k_{\mathrm{ID}}$ corresponding to ID. The user key generation algorithm UserKeyGen takes params and ID as input, and outputs a user public key $u p k_{\mathrm{ID}}$ and a user secret key $u s k_{\mathrm{ID}}$ corresponding to ID. The release algorithm Release takes $t p k$, tsk and a time period $T$ as inputs, and outputs a time signal $d_{T}$. The encryption algorithm Encrypt takes params, $t p k$, ID, $u p k_{\mathrm{ID}}, T$ and a message $m$ as inputs, and outputs a ciphertext $c$. The decryption algorithm Decrypt takes as inputs params, tpk, psk $k_{\mathrm{ID}}, u s k_{\mathrm{ID}}, d_{T}$ and a ciphertext $c^{\prime}$, and outputs the plaintext $m^{\prime}$ or $\perp$. These algorithms are assumed to satisfy that Decrypt (params, tpk, psk $k_{\mathrm{ID}}, u s k_{\mathrm{ID}}, d_{T}$, Encrypt $\left(\right.$ params, $\left.\left.t p k, \mathrm{ID}, u p k_{\mathrm{ID}}, T, m\right)\right)=m$ holds for any $m$, if $(t p k, t s k)=$ TS_Setup $(\lambda),($ params,$m s k)=\mathrm{KGC}$ _Setup $(\lambda)$, psk $_{\mathrm{ID}}=$ PartialKeyGen $($ params, $m s k, \mathrm{ID}),\left(u p k_{\mathrm{ID}}, u s k_{\mathrm{ID}}\right)=$ UserKeyGen (params, ID) and $d_{T}=$ TR.Release $(t p k, t s k, T)$ hold.

\section{A. Security}

We can consider security against $\mathrm{KGC}$, TS, receiver or outsider. Since the TS security is implied by KGC security, we present the three kinds of security.

1) Mal.KGC Security: We introduce a security notion for TRCLE: indistinguishability against adaptive identity and chosen ciphertext attacks by key generation center (Mal.KGC) security. This security ensures that a malicious key generation center, who has a master secret key msk, cannot obtain any information of message from a ciphertext without a user secret

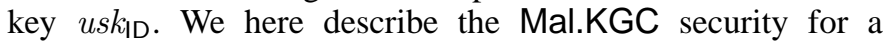
TRCLE scheme $\mathcal{T} \mathcal{R C} \mathcal{L} \mathcal{E}$ based on the following Mal.KGC game between a challenger $\mathcal{C}$ and adversary $\mathcal{A}$.

Setup

$\mathcal{C}$ runs $(t p k, t s k) \leftarrow$ TS_Setup $(\lambda)$ and sends $(\lambda, t p k, t s k)$ to $\mathcal{A}$. $\mathcal{A}$ runs (params, msk) $\leftarrow$ $\operatorname{KGC}$ Setup $(\lambda)$ and sends params to $\mathcal{C}$. $\mathcal{C}$ creates an empty list List.

Phase1

$\mathcal{A}$ can adaptively issue the following four queries.

Create User query

$\mathcal{A}$ sends (ID, $\left.p s k_{\mathrm{ID}}\right)$ to $\mathcal{C}$. When ID is in List, $\mathcal{C}$ returns $u p k_{\mathrm{ID}}$ corresponding to ID. When ID is not in List, $\mathcal{C}$ runs $\left(u p k_{\mathrm{ID}}, u s k_{\mathrm{ID}}\right)$ $\leftarrow$ UserKeyGen(params, ID) and stores $\left(\mathrm{ID}, p s k_{\mathrm{ID}}, u p k_{\mathrm{ID}}, u s k_{\mathrm{ID}}\right)$ in List. $\mathcal{C}$ returns $u p k_{\mathrm{ID}}$ corresponding to ID.

Reveal Secret Key query

$\mathcal{A}$ sends ID to $\mathcal{C}$. When ID is in List, $\mathcal{C}$ returns $u s k_{\mathrm{ID}}$ corresponding to ID. When ID is not in List, $\mathcal{C}$ returns $\perp$

Replace query

$\mathcal{A}$ sends (ID, upk', usk') to $\mathcal{C}$. When ID is in List, $\mathcal{C}$ replaces (ID, $\left.p s k_{\mathrm{ID}}, u p k_{\mathrm{ID}}, u s k_{\mathrm{ID}}\right)$ with $\left(\mathrm{ID}, p s k_{\mathrm{ID}}, u p k^{\prime}, u s k^{\prime}\right)$. If $u s k^{\prime}=\perp, \mathcal{C}$ sets $u s k^{\prime}=u s k_{\mathrm{ID}}$ When ID is not in List, $\mathcal{C}$ do nothing.

Decrypt query

$\mathcal{A}$ sends (ID, $T, c)$ to $\mathcal{C}$. When ID is in List, $\mathcal{C}$ runs $d_{T} \leftarrow$ Release $(t p k, t s k, T)$ and $m \leftarrow$ Decrypt (params, tpk, psk $\left.k_{\mathrm{ID}}, u s k_{\mathrm{ID}}, d_{T}, c\right)$ and returns $m$. When ID is not in List, $\mathcal{C}$ returns $\perp$.

\section{Challenge}

$\mathcal{A}$ sends two messages $m_{0}, m_{1}$ such that $\left|m_{0}\right|=$ $\left|m_{1}\right|$, an identity to be challenged ID* and a time period $T^{*}$ to $\mathcal{C}$. The challenge identity ID* must differ from any ID issued as Replace 
Phase2

queries in Phase1. $\mathcal{C}$ randomly chooses $b \in$ $\{0,1\}$ and sends a challenge ciphertext $c^{*}=$ Encrypt (params, tpk, ID*, upk $\left.k_{\mathrm{ID}^{*}}, T^{*}, m_{b}\right)$ to $\mathcal{A}$.

$\mathcal{A}$ can adaptively issue the above four queries in the same way as Phase1 except that the Replace queries ID must differ from the challenge identity $\mathrm{ID}^{*}$, and the decryption queries (ID, $\left.T, c\right)$ must differ from the tuple $\left(\mathrm{ID}^{*}, T^{*}, c^{*}\right)$.

\section{Guess}

$\mathcal{A}$ outputs a guess $b^{\prime} \in\{0,1\}$ and wins if $b=b^{\prime}$.

We define an advantage of $\mathcal{A}$ in the Mal.KGC game as $A d v_{\mathcal{T} \mathcal{R} \mathcal{L} \mathcal{L} \mathcal{E}, \mathcal{A}}^{\mathrm{Mal}}(\lambda)=\left|\operatorname{Pr}\left[b=b^{\prime}\right]-\frac{1}{2}\right|$, in which the probability is taken over the random coins used by $\mathcal{C}$ and $\mathcal{A}$. We say that the TRCLE scheme $\mathcal{T} \mathcal{R C} \mathcal{L} \mathcal{E}$ is Mal.KGC secure if, for any probabilistic polynomial-time adversary $\mathcal{A}$, the function $A d v_{\mathcal{T} \mathcal{R} \mathcal{C} \mathcal{L} \mathcal{E}, \mathcal{A}}^{\text {Mal }}(\lambda)$ is negligible in $\lambda$.

2) Mal.Receiver Security: We introduce a security notion for TRCLE: indistinguishability against adaptive identity and chosen ciphertext attacks by receiver (Mal.Receiver) security. This security ensures that a malicious receiver, who has a partial secret key $p s k_{\mathrm{ID}}$ and user secret key $u s k_{\mathrm{ID}}$, cannot obtain any information of message from a ciphertext without a time signal $d_{T}$ corresponding to the time designated by the sender. We here describe the Mal.Receiver security for a TRCLE scheme $\mathcal{T} \mathcal{R C} \mathcal{L} \mathcal{E}$ based on the following Mal.Receiver game between a challenger $\mathcal{C}$ and adversary $\mathcal{A}$.

Setup

Phase1

$\mathcal{C}$ runs $($ params, msk $) \leftarrow$ KGC_Setup $(\lambda),(t p k, t s k)$ $\leftarrow$ TS_Setup $(\lambda)$, and sends $(\lambda, t p k, t s k)$ to $\mathcal{A}$. $\mathcal{C}$ creates an empty list List.

$\mathcal{A}$ can adaptively issue the following five queries.

Create User query

$\mathcal{A}$ sends (ID, upk $\left.k_{\mathrm{ID}}, u s k_{\mathrm{ID}}\right)$ to $\mathcal{C}$. When ID is in List, $\mathcal{C}$ do nothing. When ID is not in List, $\mathcal{C}$ runs $p s k_{\mathrm{ID}} \leftarrow$ PartialKeyGen $($ params, $m s k$, ID $)$ and stores (ID, $\left.p s k_{\mathrm{ID}}, u p k_{\mathrm{ID}}, u s k_{\mathrm{ID}}\right)$ in List.

Reveal Partial Key query

$\mathcal{A}$ sends ID to $\mathcal{C}$. When ID is in List, $\mathcal{C}$ returns psk $k_{\mathrm{ID}}$ corresponding to ID. When ID is not in List, $\mathcal{C}$ returns $\perp$

Replace query

$\mathcal{A}$ sends (ID, upk $\left.k^{\prime} u s k^{\prime}\right)$ to $\mathcal{C}$. When ID is in List, $\mathcal{C}$ replaces (ID, $p s k_{\mathrm{ID}}, u p k_{\mathrm{ID}}, u s k_{\mathrm{ID}}$ ) with $\left(\mathrm{ID}, p s k_{\mathrm{ID}}, u p k^{\prime}, u s k^{\prime}\right)$. If $u s k^{\prime}=\perp, \mathcal{C}$ sets $u s k^{\prime}=u s k_{\mathrm{ID}}$ When ID is not in List, $\mathcal{C}$ do nothing.

Release query

$\mathcal{A}$ sends $T$ to $\mathcal{C}$. $\mathcal{C}$ runs $d_{T} \leftarrow$ Release $(t p k, t s k$, T) and returns $d_{T}$.

Decrypt query

$\mathcal{A}$ sends (ID, $T, c)$ to $\mathcal{C}$. When ID is in List, $\mathcal{C}$ runs $d_{T} \leftarrow$ Release $(t p k, t s k, T)$ and $m \leftarrow$ Decrypt(params, tpk, psk $\left.k_{\mathrm{ID}}, u s k_{\mathrm{ID}}, d_{T}, c\right)$ and Challenge returns $m$. When ID is not in List, $\mathcal{C}$ returns $\perp$.

$\mathcal{A}$ sends two messages $m_{0}, m_{1}$ such that $\left|m_{0}\right|=$
Phase2

$\left|m_{1}\right|$, an identity to be challenged $\mathrm{ID}^{*}$ and a time period $T^{*}$ to $\mathcal{C}$. The time period $T^{*}$ must differ from any $T$ issued as Release queries in Phase1. $\mathcal{C}$ randomly chooses $b \in$ $\{0,1\}$ and sends a challenge ciphertext $c^{*}=$ Encrypt (params, tpk, ID*, upk $\left.\mathrm{ID}_{\mathrm{ID}^{*}}, T^{*}, m_{b}\right)$ to $\mathcal{A}$.

$\mathcal{A}$ can adaptively issue the above five queries in the same way as Phase1 except that the Release queries $T$ must differ from $T^{*}$, and the decryption queries (ID, $T, c)$ must differ from the tuple $\left(\mathrm{ID}^{*}, T^{*}, c^{*}\right)$.

\section{Guess}

$\mathcal{A}$ outputs a guess $b^{\prime} \in\{0,1\}$ and wins if $b=b^{\prime}$.

We define an advantage of $\mathcal{A}$ in the Mal.Receiver game as $A d v_{\mathcal{T} \mathcal{R} \mathcal{C} \mathcal{L} \mathcal{E}, \mathcal{A}}^{\text {Mal }}(\lambda)=\left|\operatorname{Pr}\left[b=b^{\prime}\right]-\frac{1}{2}\right|$, in which the probability is taken over the random coins used by $\mathcal{C}$ and $\mathcal{A}$. We say that the TRCLE scheme $\mathcal{T} \mathcal{R C} \mathcal{L E}$ is Mal.Receiver secure if, for any probabilistic polynomial-time adversary $\mathcal{A}$, the function $\operatorname{Adv} v_{\mathcal{T} \mathcal{R} \mathcal{C} \mathcal{L} \text { E. } \mathcal{A}}^{\text {Mal. }}(\lambda)$ is negligible in $\lambda$.

3) Outsider Security: We introduce a security notion for TRCLE: indistinguishability against adaptive identity and chosen ciphertext attacks by outsider (Outsider) security. This security ensures that a outsider, who has a public parameter params and tpk, cannot obtain any information of message from a ciphertext without user secret key $u s k_{\mathrm{ID}}$. We here describe the Outsider security for a TRCLE scheme $\mathcal{T} \mathcal{R C} \mathcal{L} \mathcal{E}$ based on the following Outsider game between a challenger $\mathcal{C}$ and adversary $\mathcal{A}$.

Setup

$\mathcal{C}$ runs $($ params, $m s k) \leftarrow \mathrm{KGC} \operatorname{Setup}(\lambda),(t p k, t s k)$ $\leftarrow$ TS_Setup $(\lambda)$, and sends (params, tpk) to $\mathcal{A}$. $\mathcal{C}$ creates an empty list List.

Phase1

$\mathcal{A}$ can adaptively issue the following six queries.

Create User query

$\mathcal{A}$ sends ID to $\mathcal{C}$. When ID is in List, $\mathcal{C}$ returns $u p k_{\mathrm{ID}}$ corresponding to ID. When ID is not in List, $\mathcal{C}$ runs $p s k_{\mathrm{ID}} \leftarrow$ PartialKeyGen (params, msk, ID) and (upk $k_{\mathrm{ID}}$, $\left.u s k_{\mathrm{ID}}\right) \leftarrow$ UserKeyGen $($ params, ID), and stores $\left(\mathrm{ID}, p s k_{\mathrm{ID}}, u p k_{\mathrm{ID}}, u s k_{\mathrm{ID}}\right)$ in List. $\mathcal{C}$ returns $u p k_{\mathrm{ID}}$ corresponding to ID.

Reveal Partial Key query

$\mathcal{A}$ sends ID to $\mathcal{C}$. When ID is in List, $\mathcal{C}$ returns $p s k_{\mathrm{ID}}$ corresponding to ID. When ID is not in List, $\mathcal{C}$ returns $\perp$

Reveal Secret Key query

$\mathcal{A}$ sends ID to $\mathcal{C}$. When ID is in List, $\mathcal{C}$ returns $u s k_{\mathrm{ID}}$ corresponding to ID. When ID is not in List, $\mathcal{C}$ returns $\perp$

Replace query

$\mathcal{A}$ sends (ID, upk $k^{\prime}, u s k^{\prime}$ ) to $\mathcal{C}$. When ID is in List, $\mathcal{C}$ replaces (ID, $p s k_{\mathrm{ID}}, u p k_{\mathrm{ID}}, u s k_{\mathrm{ID}}$ ) with $\left(\mathrm{ID}, p s k_{\mathrm{ID}}, u p k^{\prime}, u s k^{\prime}\right)$. If $u s k^{\prime}=\perp, \mathcal{C}$ sets $u s k^{\prime}=u s k_{\mathrm{ID}}$ When ID is not in List, $\mathcal{C}$ do nothing.

Release query 
$\mathcal{A}$ sends $T$ to $\mathcal{C}$. $\mathcal{C}$ runs $d_{T} \leftarrow$ Release $(t p k, t s k$, T) and returns $d_{T}$.

Decrypt query

$\mathcal{A}$ sends (ID, $T, c)$ to $\mathcal{C}$. When ID is in List, $\mathcal{C}$ runs $d_{T} \leftarrow$ Release $(t p k, t s k, T)$ and $m \leftarrow$ $\operatorname{Decrypt}\left(\right.$ params, tpk, psk $\left.k_{\mathrm{ID}}, u s k_{\mathrm{ID}}, d_{T}, c\right)$ and reChallenge turns $m$. When ID is not in List, $\mathcal{C}$ returns $\perp$.

$\mathcal{A}$ sends two messages $m_{0}, m_{1}$ such that $\left|m_{0}\right|=$ $\left|m_{1}\right|$, an identity to be challenged ID $^{*}$ and a time period $T^{*}$ to $\mathcal{C}$. The challenge identity ID* must differ from any ID issued as Reveal Partial Key queries in Phase1. $\mathcal{C}$ randomly chooses $b \in\{0,1\}$ and sends a challenge ciphertext $c^{*}=$ Encrypt $\left(\right.$ params, $\left.t p k, \mathrm{ID}^{*}, u p k_{\mathrm{ID}^{*}}, T^{*}, m_{b}\right)$ to $\mathcal{A}$.

Phase2

$\mathcal{A}$ can adaptively issue the above six queries in the same way as Phase1 except that the Reveal Secret Key queries ID must differ from ID*, and the decryption queries (ID, $T, c)$ must differ from the tuple $\left(\mathrm{ID}^{*}, T^{*}, c^{*}\right)$.

Guess

$\mathcal{A}$ outputs a guess $b^{\prime} \in\{0,1\}$ and wins if $b=b^{\prime}$.

We define an advantage of $\mathcal{A}$ in the Outsider game as $A d v_{\mathcal{T} \mathcal{R} \mathcal{C} \mathcal{L} \mathcal{E}, \mathcal{A}}(\lambda)=\left|\operatorname{Pr}\left[b=b^{\prime}\right]-\frac{1}{2}\right|$, in which the probability is taken over the random coins used by $\mathcal{C}$ and $\mathcal{A}$. We say that the TRCLE scheme $\mathcal{T} \mathcal{R C} \mathcal{L} \mathcal{E}$ is Outsider secure if, for any probabilistic polynomial-time adversary $\mathcal{A}$, the function $A d v_{\mathcal{T} \mathcal{R} \mathcal{C} \mathcal{L E}, \mathcal{A}}^{\text {Outsider }}(\lambda)$ is negligible in $\lambda$.

\section{CONSTRUCTION}

Here we present a generic construction of TRCLE scheme from PKE scheme, IBE scheme and one-time signature scheme.

\section{A. Construction}

Let $\Delta=$ (PKE.Gen, PKE.Enc, PKE.Dec) be a publickey encryption scheme, $\Pi=($ IBE.Setup, IBE.Ext, IBE.Enc, IBE.Dec) and $\Pi^{\prime}=\left(\mathrm{IBE}^{\prime}\right.$.Setup, IBE $\mathrm{IBX}^{\prime}$.Ex, IBE'.Enc, $\mathrm{IBE}^{\prime}$.Dec) be identity-based encryption schemes, and $\Sigma=$ (SigGen, Sign, Verify) be a one-time signature scheme.

A TRCLE scheme $\Gamma=$ (KGC_Setup, TS_Setup, PartialKeyGen, UserKeyGen, Release, Encrypt, Decrypt) is constructed as follows.

$\operatorname{KGC} \operatorname{Setup}(\lambda)$ :

Step 1: Run IBE.Setup on input $\lambda$ to generate (params, $m s k$ ).

Step 2: Return (params, msk).

TS_Setup $(\lambda)$ :

Step 1: Run IBE'.Setup on input $\lambda$ to generate (params', $\left.m s k^{\prime}\right)$.

Step 2: Set $t p k=$ params $^{\prime}$ and $t s k=m s k^{\prime}$.

Step 3: Return $(t p k, t s k)$.

PartialKeyGen(params, msk, ID):

Step 1: Run IBE.Ext (params, msk, ID) to obtain $d_{I D}$.

Step 2: Return $d_{I D}$.

UserKeyGen(params, ID):
Step 1: Run PKE.Gen on input $\lambda$ to generate $(p k, s k)$.

Step 2: Set $u p k_{I D}=p k$ and $u s k_{\mathrm{ID}}=s k$.

Step 3: Return $\left(u p k_{I D}, u s k_{I D}\right)$.

Release $(t p k, t s k, T)$ :

Step 1: Run IBE'. $\operatorname{Ext}(t p k, t s k, T)$ to obtain $d_{T}$.

Step 2: Return $d_{T}$.

Encrypt (params, tpk, ID, upk $\left.{ }_{I D}, T, m\right)$ :

Step 1: Run SigGen on input $\lambda$ to generate $(s k, v k)$.

Step 2: Randomly choose $s_{1} \in\{0,1\}^{|m|}$ and $s_{2} \in\{0,1\}^{|m|}$.

Step 3: Compute $s_{3}=s_{1} \oplus s_{2} \oplus m$.

Step 4: Compute $c_{1}=\mathrm{IBE}$.Enc(params, ID, $s_{1} \| v k$ ).

Step 5: Compute $c_{2}=\mid \mathrm{BE}^{\prime}$. $\operatorname{Enc}\left(t p k, T, s_{2} \| v k\right)$.

Step 6: Compute $c_{3}=\operatorname{PKE}$.Enc $\left(u p k_{I D}, s_{3} \| v k\right)$.

Step 7: Compute $\sigma=\operatorname{Sign}\left(s k, c_{1}\left\|c_{2}\right\| c_{3}\|I D\| T\right)$.

Step 8: Set $c=\left(c_{1}, c_{2}, c_{3}, I D, T, v k, \sigma\right)$.

Step 9: Return $c$.

Decrypt (params, tpk, psk $\left.{ }_{I D}, u s k_{I D}, d_{T}, c\right)$ :

Step 1: Parse $c$ as $c=\left(c_{1}, c_{2}, c_{3}, I D, T, v k, \sigma\right)$.

Step 2: If Verify $\left(v k, c_{1}\left\|c_{2}\right\| c_{3}\|I D\| T, \sigma\right)=$ reject, then return $\perp$ and stop.

Step 3: Compute $s_{1} \| v k^{\prime}=$ IBE.Dec $\left(\right.$ params, $\left.p s k_{I D}, c_{1}\right)$.

Step 4: Compute $s_{2} \| v k^{\prime \prime}=\mathrm{IBE}^{\prime} \cdot \operatorname{Dec}\left(t p k, d_{T}, c_{2}\right)$.

Step 5: Compute $s_{3} \| v k^{\prime \prime \prime}=\operatorname{PKE} \cdot \operatorname{Dec}\left(u s k_{I D}, c_{3}\right)$.

Step 6: If $v k=v k^{\prime}=v k^{\prime \prime}=v k^{\prime \prime \prime}$, then return $m=$ $s_{1} \oplus s_{2} \oplus s_{3}$ else return $\perp$.

\section{B. Security of TRCLE}

1) Mal.KGC security:

Theorem 1: If $\Delta$ is an IND-CCA secure public-key encryption scheme and $\Sigma$ is an OT-sEUF-CMA secure one-time signature scheme, then $\Gamma$ is a Mal.KGC secure timed-release certificateless encryption scheme.

Proof(Theorem 1) Suppose $\mathcal{A}$ is an adversary that breaks the Mal.KGC security of $\Gamma$. We construct a simulator $\mathcal{B}$ which breaks the IND-CCA security of the PKE scheme $\Delta$ using $\mathcal{A}$. Here we say a ciphertext $c=\left(c_{1}, c_{2}, c_{3}, \mathrm{ID}, T, v k, \sigma\right)$ is valid if $\operatorname{Verify}\left(v k, c_{1}\left\|c_{2}\right\| c_{3}\|\mathrm{ID}\| T, \sigma\right)=$ accept. Let $c^{*}=\left(c_{1}^{*}, c_{2}^{*}, c_{3}^{*}, \mathrm{ID}^{*}, T^{*}, v k^{*}, \sigma^{*}\right)$ be the challenge ciphertext. Let Forge denote the event that $\mathcal{A}$ submits a valid ciphertext $c=\left(c_{1}, c_{2}, c_{3}, T, \mathrm{ID}, v k^{*}, \sigma\right)$ as a Decrypt query to $\mathcal{C}$ in the Phase2, and Succ denote the event that $\mathcal{A}$ wins the Mal.KGC game. We assume that $\mathcal{A}$ issues at most $q$ distinct Create User queries. We prove the following claims.

Claim 1: $\operatorname{Pr}[$ Forge $]$ is negligible. ligible.

Claim 2: $\mid \operatorname{Pr}[$ Succ $\wedge \overline{\text { Forge }}]+\frac{1}{2} \operatorname{Pr}[$ Forge $]-\frac{1}{2} \mid$ is neg-

Proof(Claim 1) We assume Forge occurs. Then, we construct a forger $\mathcal{F}$ who breaks OT-sEUF-CMA security of the onetime signature scheme $\Sigma$, from $\mathcal{A}$. The description of $\mathcal{F}$ is as follows.

Setup

$\mathcal{F}$ receives $v k^{*}$ from $\mathcal{C} . \mathcal{F}$ runs $(t p k, t s k) \leftarrow$ TS_Setup $(\lambda)$ and sends $(\lambda, t p k, t s k)$ to $\mathcal{A}$.

Query

$\mathcal{F}$ responds $\mathcal{A}$ 's four queries as the challenger in the Mal.KGC game. If $\mathcal{A}$ happens to issue a valid ciphertext $c=\left(c_{1}, c_{2}, c_{3}, \mathrm{ID}, T, v k^{*}, \sigma\right)$ 


\section{Challenge}

as decryption query to $\mathcal{F}$ before Challenge in the Mal.KGC game, then $\mathcal{F}$ simply outputs $\left(c_{1}\left\|c_{2}\right\| c_{3} \| T|| \mathrm{ID}, \sigma\right)$ as forgery and stops.

If $\mathcal{A}$ outputs $\left(m_{0}, m_{1}, \mathrm{ID}^{*}, T^{*}\right)$ as challenge, $\mathcal{F}$ randomly chooses $s_{1} \in\{0,1\}^{|m|}, s_{2} \in\{0,1\}^{|m|}$ and $b \in\{0,1\}$, and computes $s_{3}=s_{1} \oplus s_{2} \oplus$ $m_{b}$. Then $\mathcal{F}$ computes $c_{1}^{*}=$ IBE. Enc (params, ID*, $\left.s_{1} \| v k^{*}\right), c_{2}^{*}=\mathrm{IBE}^{\prime}$.Enc $\left(t p k, T^{*}, s_{2} \| v k^{*}\right)$ and $c_{3}^{*}$ $=$ PKE.Enc $\left(p u b_{\mid \mathrm{DD}^{*}}, s_{3} \| v k^{*}\right)$, then issues $m^{*}=$ $\left(c_{1}\left\|c_{2}\right\| c_{3}\left\|\mid \mathrm{ID}^{*}\right\| T^{*}\right)$ as signing query to $\mathcal{C}$ and obtains $\sigma^{*}$. Finally $\mathcal{F}$ returns $c^{*}=\left(c_{1}^{*}, c_{2}^{*}, c_{3}^{*}\right.$, $\left.\mathrm{ID}^{*}, T^{*}, v k^{*}, \sigma^{*}\right)$ as the challenge ciphertext to $\mathcal{A}$.

Forge

If $\mathcal{A}$ issues a valid ciphertxt $c=\left(c_{1}, c_{2}, c_{3}\right.$, ID, $\left.T, v k^{*}, \sigma\right)$ as decryption query, then $\mathcal{F}$ outputs $\left(c_{1}\left\|c_{2}\right\| c_{3}\|\mathrm{ID}\| T, \sigma\right)$ as forgery.

$\mathcal{F}$ can forge the signature if $\mathcal{A}$ issues a decryption query that causes the event Forge. It, however, contradicts that $\Sigma$ is OT-sEUF-CMA secure. Thus, $\operatorname{Pr}$ [Forge] is negligible.

Proof(Claim 2) We construct an adversary $\mathcal{B}$ who breaks INDCCA security of the PKE scheme $\Delta$ using $\mathcal{A}$. The description of $\mathcal{B}$ is as follows.

Setup

$\mathcal{B}$ receives $p k^{*}$ from $\mathcal{C}$. Then $\mathcal{B}$ runs $(t p k, t s k)$ $\leftarrow$ TS_Setup $(\lambda)$ and sends $(\lambda, t p k, t s k)$ to $\mathcal{A}$ and randomly chooses index $i \in\{1,2, \cdots q\}$. B creates an empty list List.

Phase1

The response of $\mathcal{B}$ for $\mathcal{A}$ 's queries is as follows.

Create User

When a given query (ID, $\left.p s k_{\mathrm{ID}}\right)$ is the $i$-th Create User query, $\mathcal{B}$ stores $\left(I D, p s k_{\mathrm{ID}}, p k^{*}, \perp\right)$ into List and returns $p k^{*}$ as $u p k_{\mathrm{ID}}$. When it is not the $i$-th, $\mathcal{B}$ runs $\left(u p k_{\mathrm{ID}}, u s k_{\mathrm{ID}}\right) \leftarrow$ UserKeyGen(params, ID). Then $\mathcal{B}$ stores (ID, $\left.p s k_{\mathrm{ID}}, u p k_{\mathrm{ID}}, u s k_{\mathrm{ID}}\right)$ into List and returns $u p k_{\mathrm{ID}}$.

\section{Reveal Secret Key}

When ID is in List, if $u p k_{\mathrm{ID}}=p k^{*}, \mathcal{B}$ stops and outputs random bit $b^{\prime}$ and otherwise returns $u s k_{\mathrm{ID}}$. When ID is not in List, $\mathcal{B}$ returns $\perp$.

Replace

When ID is in List, if $u p k_{\mathrm{ID}}=p k^{*}, \mathcal{B}$ stops and outputs random bit $b^{\prime}$. If $u s k^{\prime}=\perp, \mathcal{B}$ sets $u s k^{\prime}$ $=u s k_{\mathrm{ID}}$.Then it replaces (ID, $\left.p s k_{\mathrm{ID}}, u p k_{\mathrm{ID}}, u s k_{\mathrm{ID}}\right)$ with (ID, $\left.p s k_{\mathrm{ID}}, u p k^{\prime}, u s k^{\prime}\right)$.

Decrypt

When ID is in List, if $u p k_{\mathrm{ID}} \neq p k^{*}, \mathcal{B}$ runs $d_{T} \leftarrow$ Release $(t p k, t s k, T)$ and $m \leftarrow$ Decrypt(params, tpk, psk $\left.k_{\mathrm{ID}}, u s k_{\mathrm{ID}}, d_{T}, c\right)$ and returns $m$. If upk $k_{\mathrm{ID}}=p k^{*}, \mathcal{B}$ responds as follows. If Verify $\left(v k, c_{1}\left\|c_{2}\right\| c_{3} \|\right.$ ID $\left.\| T, \sigma\right)=$ reject, then $\mathcal{B}$ returns $\perp$ to $\mathcal{A}$. Otherwise $\mathcal{B}$ runs $s_{1} \| v k^{\prime} \leftarrow \operatorname{IBE} . \operatorname{Dec}\left(\right.$ params, $\left.d_{\mathrm{ID}}, c_{1}\right) s_{2} \| v k^{\prime \prime} \leftarrow$
$\mathrm{IBE}^{\prime} \cdot \operatorname{Dec}\left(t p k, d_{T}, c_{2},\right)$ and issues decryption query $c_{3}$ to $\mathcal{C}$ and obtains $s_{3} \| v k^{\prime \prime \prime} . \mathcal{B}$ returns $m$ $=s_{1} \oplus s_{2} \oplus s_{3}$ to $\mathcal{A}$ if $v k=v k^{\prime}=v k^{\prime \prime}$, and otherwise $\mathcal{B}$ returns $\perp$ to $\mathcal{A}$. When ID is not in List, $\mathcal{B}$ returns $\perp$ to $\mathcal{A}$.

\section{Challenge}

If $\mathcal{A}$ outputs $\left(m_{0}, m_{1}, \mathrm{ID}^{*}, T^{*}\right)$ as challenge, $\mathcal{B}$ runs $\left(s k^{*}, v k^{*}\right) \leftarrow \operatorname{SigGen}(\lambda)$ and randomly chooses $s_{1} \in\{0,1\}^{|m|}$ and $s_{2} \in\{0,1\}^{|m|}$ and runs $c_{1}^{*}=\mathrm{IBE} . \operatorname{Enc}\left(\right.$ params, $\left.\mathrm{ID}^{*}, s_{1} \| v k^{*}\right)$ and $c_{2}^{*}=\mathrm{IBE}^{\prime}$.Enc $\left(t p k, T^{*}, s_{2} \| v k^{*}\right)$. Then $\mathcal{B}$ computes $M_{0}=\left[\left(s_{1} \oplus s_{2} \oplus m_{0}\right) \| v k^{*}\right]$ and $M_{1}=\left[\left(s_{1} \oplus s_{2} \oplus m_{1}\right) \| v k^{*}\right]$, and issues $\left(M_{0}, M_{1}\right)$ as $\mathcal{B}$ 's challenge to $\mathcal{C}$ and obtains a ciphertext $c_{3}^{*}$. $\mathcal{B}$ runs $\sigma^{*}=\operatorname{Sign}\left(s k^{*}, c_{1}^{*}\left\|c_{2}^{*}\right\| c_{3}^{*}\left\|\mathrm{ID}^{*}\right\| T^{*}\right)$ and returns $c^{*}=\left(c_{1}^{*}, c_{2}^{*}, c_{3}^{*}, \mathrm{ID}^{*}, T^{*}, v k^{*}, \sigma^{*}\right)$ as challenge ciphertext to $\mathcal{A}$.

Phase2

$\mathcal{B}$ responds to Create User query, Reveal Secret Key query and Replace query in the same way as in Phase1. $\mathcal{B}$ responds to Decrypt query as follows.The followings are done in a sequential way.

Step1

If $\operatorname{Verify}\left(v k, c_{1}\left\|c_{2}\right\| c_{3}\|\mathrm{ID}\| T \|, \sigma\right)=$ reject, then $\mathcal{B}$ returns $\perp$ and skips step2 4 .

Step2

If $v k=v k^{*}$, then $\mathcal{B}$ stops and outputs random bit $b^{\prime}$.

Step3

Step4

$$
\text { If } c_{3}=c_{3}^{*} \text {, then } \mathcal{B} \text { returns } \perp \text {. }
$$

$\mathcal{B}$ responds in the same way as in Phase1.

Guess If $\mathcal{A}$ outputs a bit, then $\mathcal{B}$ outputs the same bit as its guess.

We consider the $\mathcal{B}$ 's simulation of the response to decryption queries in Phase2. In the case of Verify $=$ reject in Step1, $\mathcal{B}$ returns $\perp$ in the same way as in our Decrypt algorithm, and then it perfectly simulates the challenger in Mal.KGC game. In the case of $v k=v k^{*}$ in Step2, the event Forge occurs. In the case of $c_{3}=c_{3}^{*}$ in Step3, since $c_{3}$ equals to $c_{3}^{*}$, the decryption of $c_{3}$ is $M_{0}=\left[\left(s_{1} \oplus s_{2} \oplus m_{0}\right) \| v k^{*}\right]$ or $M_{1}=\left[\left(s_{1} \oplus s_{2} \oplus m_{1}\right) \| v k^{*}\right]$. However, since $v k \neq v k^{*}$, the decryption of $c$ is $\perp$, and then $\mathcal{B}$ simulates perfectly. In the case of $c_{3} \neq c_{3}^{*}, \mathcal{B}$ can issue the valid decryption query $c_{3}$ to C.

If the event Forge does not occurs, $\mathcal{B}$ perfectly simulates the challengers in the IND-ID-CCA denote the event that $\mathcal{B}$ wins the IND-CCA game.

We see that

$$
\begin{aligned}
A d v_{\Delta, \mathcal{B}}^{\mathrm{IND}-\mathrm{CCA}}= & \left|\operatorname{Pr}\left[\operatorname{Succ}^{\mathcal{P K E}}\right]-\frac{1}{2}\right| \\
\geq & \mid \frac{1}{2} \cdot\left(1-\frac{1}{q}\right) \\
& +\left(\operatorname{Pr}\left[\operatorname{Succ} \wedge \overline{\text { Forge }}+\frac{1}{2} \operatorname{Pr}[\text { Forge }]\right) \cdot \frac{1}{q}-\frac{1}{2} \mid\right. \\
& \quad 283 \mid \mathrm{P} \text { a g e }
\end{aligned}
$$




$$
=\mid \operatorname{Pr}\left[\text { Succ } \wedge \overline{\text { Forge }}+\frac{1}{2} \operatorname{Pr}[\text { Forge }]-\frac{1}{2} \mid \cdot \frac{1}{q}\right. \text {. }
$$

$A d v_{\Delta, \mathcal{B}}^{\mathrm{IND}-\mathrm{CCA}}$ is negligible since we assume $\Delta$ is IND-CCA secure. Therefore, $\mid \operatorname{Pr}\left[\operatorname{Succ} \wedge \overline{\text { Forge }}+\frac{1}{2} \operatorname{Pr}[\right.$ Forge $]-\frac{1}{2} \mid$ is also negligible.

We see that

$$
\begin{aligned}
A d v_{\Gamma, \mathcal{A}}^{\text {Mal.KGC }}= & \mid \operatorname{Pr}[\text { Succ }]-\frac{1}{2} \mid \\
= & \mid \operatorname{Pr}[\text { Succ } \wedge \text { Forge }]-\frac{1}{2} \operatorname{Pr}[\text { Forge }] \\
& +\frac{1}{2} \operatorname{Pr}[\text { Forge }]+\operatorname{Pr}\left[\text { Succ } \wedge \overline{\text { Forge }]}-\frac{1}{2} \mid\right. \\
\leq & \mid \operatorname{Pr}[\text { Succ } \wedge \text { Forge }]-\frac{1}{2} \operatorname{Pr}[\text { Forge }] \mid \\
& +\mid \operatorname{Pr}\left[\text { Succ } \wedge \overline{\text { Forge }]}+\frac{1}{2} \operatorname{Pr}[\text { Forge }]-\frac{1}{2} \mid\right. \\
\leq & \frac{1}{2} \operatorname{Pr}[\text { Forge }] \\
& +\mid \operatorname{Pr}\left[\text { Succ } \wedge \overline{\text { Forge }]}+\frac{1}{2} \operatorname{Pr}[\text { Forge }]-\frac{1}{2} \mid .\right.
\end{aligned}
$$

$A d v_{\Gamma, \mathcal{A}}^{\mathrm{Mal.KGC}}$ is negligible from Claim 1 and Claim 2. This completes the proof of Theorem 1 .

\section{2) Mal.Receiver security:}

Theorem 2: If $\Pi^{\prime}$ is an IND-ID-CCA secure identitybased encryption scheme and $\Sigma$ is an OT-sEUF-CMA secure one-time signature scheme, then $\Gamma$ is a Mal.Receiver secure timed-release certificateless encryption scheme.

The proof is almost the same as that of IND-ID-CCA $A_{C R}$ security in [3].

\section{3) Outsider security:}

Theorem 3: If $\Pi$ is an IND-ID-CCA secure identity-based encryption scheme and $\Sigma$ is an OT-sEUF-CMA secure onetime signature scheme, then $\Gamma$ is a Outsider secure timedrelease certificateless encryption scheme.

The proof is almost the same as that of IND-ID-CCA $A_{C R}$ security in [3].

\section{CONCLUSION}

In this paper, we introduced a notion of TRCLE and defined Mal.KGC security, Mal.Receiver security and Outsider security. Moreover, we showed a generic construction of TRCLE in which a constructed scheme achieves those security if the primitive PKE scheme is IND-CCA secure, the primitive IBE schemes are IND-ID-CCA secure and the primitive one-time signature scheme is OT-sEUF-CMA secure.

\section{ACKNOWLEDGMENT}

This work was supported by JSPS KAKENHI Grant Number 26330160 .

\section{REFERENCES}

[1] T. May, "Timed-release crypto," Manuscript, February 1993.

[2] A. C.-F. Chan and I. F. Blake, "Scalable, server-passive, useranonymous timed release cryptography," in ICDCS 2005. IEEE Computer Society, 2005, pp. 504-513.

[3] T. Oshikiri and T. Saito, "Timed-release identity-based encryption," IPSJ Journal, vol. 55, no. 9, pp. 1964-1970, sep 2014.

[4] - "Timed-release hierarchical identity-based encryption," International Journal of Advanced Computer Science and Applications(IJACSA), vol. 5, no. 11, pp. 148-154, 2014.

[5] Y. Dodis and J. Katz, "Chosen-ciphertext security of multiple encryption," in TCC 2005, ser. Lecture Notes in Computer Science, J. Kilian, Ed., vol. 3378. Springer-Verlag, 2005, pp. 188-209.

[6] S. S. Al-Riyami and K. G. Paterson, "Certificateless public key cryptography," in ASIACRYPT 2003, ser. Lecture Notes in Computer Science, C. S. Laih, Ed., vol. 2894. Springer-Verlag, 2003, pp. 452-473.

[7] M. Naor and M. Yung, "Public-key cryptosystems provably secure against chosen ciphertext attacks," in STOC '90. ACM, 1990, pp. 427-437.

[8] A. Shamir, "Identity-based cryptosystems and signature schemes," in CRYPTO '84, ser. Lecture Notes in Computer Science, G. R. Blakley and D. Chaum, Eds., vol. 196. Springer-Verlag, 1985, pp. 47-53.

[9] D. Boneh and M. K. Franklin, "Identity-based encryption from the Weil pairing," SIAM Journal on Computing, vol. 32, no. 3, pp. 584-615, 2003, a preliminary version appeared in CRYPTO 2001, 2001.

[10] R. C. Merkle, "A digital signature based on a conventional encryption function," in A Conference on the Theory and Applications of Cryptographic Techniques on Advances in Cryptology, ser. CRYPTO '87. London, UK, UK: Springer-Verlag, 1988, pp. 369-378. [Online]. Available: http://dl.acm.org/citation.cfm?id=646752.704751 